\title{
PRIMARY PEAK RATIO CORRELATION TO THE MEASUREMENT ACCURACY OF PIV METHOD
}

\author{
Jan NovotnÝ, Ilona Machovská*
}

\author{
Department of Fluid Dynamics and Thermodynamics, Czech Technical University in Prague, Czech Republic \\ * corresponding author: Ilona.Machovska@fs.cvut.cz
}

\begin{abstract}
An estimation of a measurement accuracy at each measured point is crucial regarding the applicability of results of the measurements. The aim of this work is to determine the correlation between individual metrics and the measurement accuracy by using corrected metrics of the correlation plane. This work is based on defining a corrected metric using known metrics corrected by the displacement measured in the last iteration, the number of the particles and the velocity gradient inside the interrogation area. The resulting tests are performed using conventional synthetic tests. The discovered dependencies between individual corrected metrics are subsequently approximated in order to determine the measurement accuracy. And, finally, the most suitable variant for the determination of the accuracy of the measurement by the particle image velocimetry method is specified.
\end{abstract}

KEYWORDS: primary peak ratio; cross-correlation; measurement accuracy; PIV method; synthetic tests.

\section{INTRODUCTION}

In this work, we focus on a description of the interconnection between the ratio of the primary and secondary peak in the correlation plane, known as Primary peak ratio (PPR), and the error of evaluation of the measured displacement by the PIV method. The aim of this work is the correction of the PPR metric by the main parameters that define the quality of the measured data in order to find a clear correlation between the metric and the measurement accuracy of the PIV method.

One option to evaluate the measurement accuracy at a specific point is to track the dependency of the Primary peak ratio. It is possible to split the cross-correlation of particle images $R_{\mathrm{S}}$ into the crosscorrelation of the noise $R_{\mathrm{C}}$, cross-correlation of the signal and noise $R_{\mathrm{F}}$ and cross-correlation of the signal $R_{\mathrm{D}}$. A brief illustration is shown in Figure 1 . Article [1, 2] state that the height of the signal peak and the PPR are proportional to the number of particles $N_{\mathrm{I}}$. Another factor that affects the height of the peak is the number of lost pairs $F_{\mathrm{I}}$. The diameter and shape of the peak are also influenced by the particle diameter Di and by the velocity gradient $F_{\Delta}$ inside the interrogation area IA. If this inequality $\left(N_{\mathrm{I}} F_{\mathrm{I}} F_{\Delta}>5\right)$ applies, there is a $95 \%$ chance that the highest peak is equal to the signal peak. The evaluation of the mentioned parameters $\left(N_{\mathrm{I}}, F_{\mathrm{I}}, F_{\Delta}\right)$ is not so trivial and that is why these parameters are not currently used to determine the measurement accuracy. Though it is difficult to evaluate the mentioned parameters, more than 30 years ago, a clear context has been found between the measurement accuracy and the shape of the correlation plane [3, 4. When using the classic PIV iterative algorithm, the measurement, where the highest peak represents the displacement and the sec- ond highest peak represents the result of the random correlation - noise peak, can be considered as the correct one.

Over the 30-year existence of the PIV method, a whole range of metrics has been defined [6, 7]. The above-mentioned metric PPR complements the ratio of quadrates of the primary peak height and the root mean square of all peak heights, known as Peak to root mean square ratio (PRMSR), and the ratio of the primary peak height quadrate and the energy in the correlation plane, known as Peak to correlation energy (PCE). To the mentioned main metrics, a metric, called the Mutual information MI, has been added. The definition of the metric MI is different from the main metrics. The metrics PPR, PRMSR and PCE are defined only by resulting the correlation plane, from which the displacement is also defined. To determine the MI metric, it is necessary to evaluate the final cross-correlation plane and, moreover, the autocorrelation plane defined by the autocorrelation of the average particle [8]. The metric MI is then defined as the ratio of maximum values in the crosscorrelation and autocorrelation plane:

$$
\mathrm{MI}=\frac{\max . \text { val. in corr. pl.(cross-corr. of all part.) }}{\text { max. val. in corr. pl.(autocorr. of av. part.) }}
$$

A schematic procedure of the calculation of the metric MI is shown in Figure 2.

This metric represents the number of particles $N_{\mathrm{I}}$ inside the interrogation area and it is a very good measure of the quality of the correlation plane. In $[8$, the synthetic tests, which have been done with the number of particles $N_{\text {I }}$ from 5 to 30 particles in an interrogation area with a size of $32 \times 32$ pixels, are very well described. Results of these synthetic tests were divided into 40 subgroups according to the value 

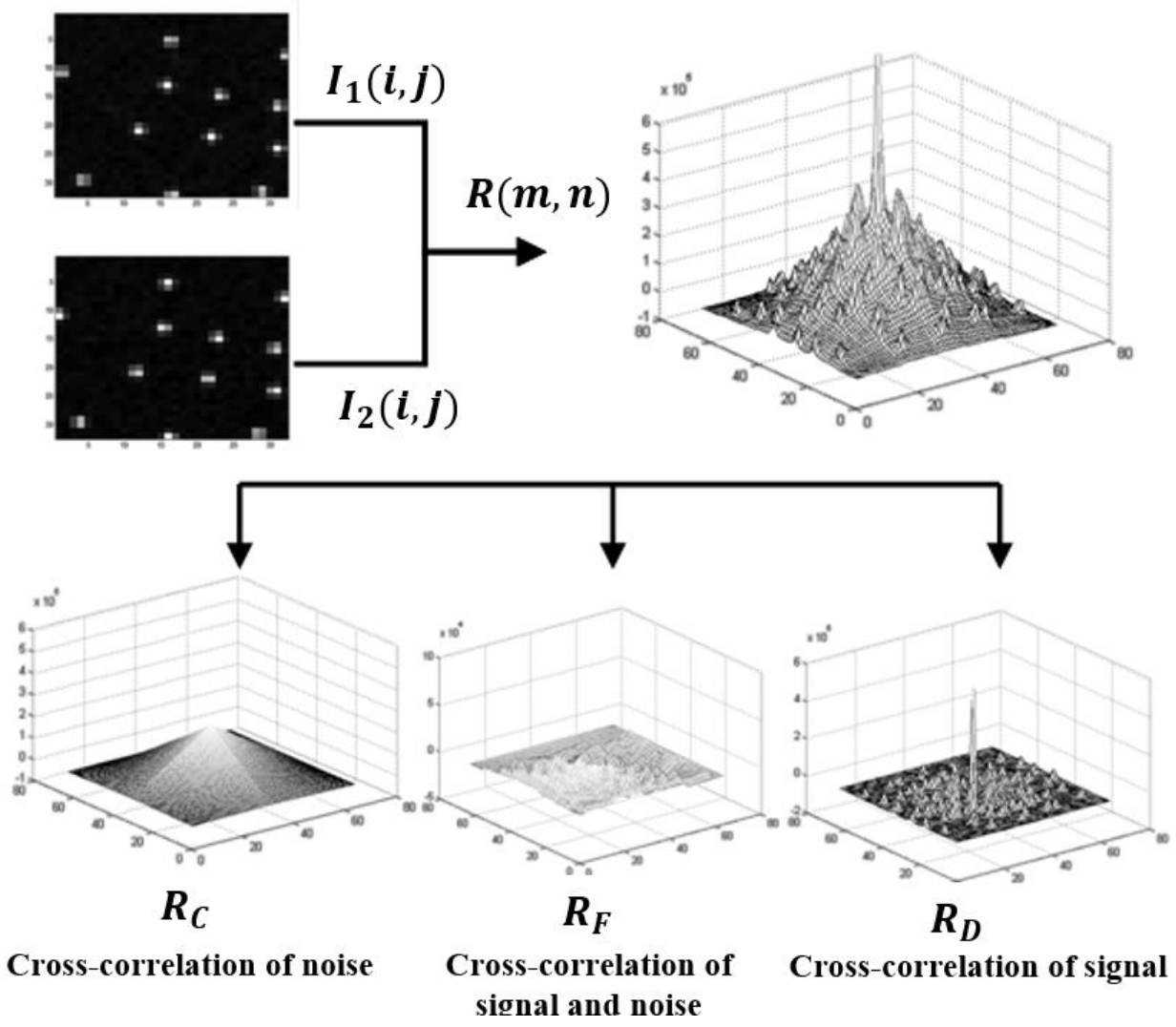

FigURE 1. The effect of individual components of the signal on the resulting shape of correlation plane; IA = $32 \times 32$ pixels, $N_{\mathrm{I}}=10, F_{\Delta}=0$ [5].

of the tested metric. For each range of the value of the metric, the error was evaluated as the root mean square error of the measurement in each subgroup. For a real use of the individual dependencies, authors of [8] also defined the approximation function of the mentioned dependencies. The following equation is based on the model, which was presented in [7], and then modified in [8] to the following form:

$$
\sigma^{2}=\left(M \exp \left(-\frac{1}{2}\left(\frac{\phi-N}{S}\right)^{2}\right)\right)^{2}+\left(A \phi^{B}\right)^{2}+C^{2} .
$$

The dependency of the evaluated root mean square error on the values of the PPR is shown in Figure 3.

The factor $M$ in 2 is proportional to the total error of the measurement and $\phi$ is the value of the adequate metric. The coefficient $\mathrm{S}$ is proportional to the real achievable value of each metric. The minimum value of $N$ can easily be derived from the definition of the appropriate metric. For the PPR, the minimum value of $N$ is $N_{\min }=1$, for the PRMSR, the minimum value of $N$ is $N_{\min }=4$, for the PCE, the minimum value of $N$ is $N_{\min }=1$. For the MI, the minimum value of $N$ is $N_{\text {min }}=0$, considering no particles inside the interrogation area. The next part of (2) approximates the effect of correctly measured data and their contribution to the total error of the measurement. The parameter $\mathrm{A}$ is proportional to the deviation from the correct value and, vice versa, the parameter $\mathrm{C}$ corresponds to the minimum deviation of the measurement.

According to [8], the total error of the measurement dependency on the PPR metric can be defined as follows:

$$
\begin{aligned}
\sigma_{\mathrm{SCC}}^{2}= & \left.10.47 \exp \left(-\frac{1}{2}\left(\frac{P P R-1}{1.12}\right)^{2}\right)\right)^{2} \\
& +\left(1.913 \phi^{-1.371}\right)^{2}+\left(2.221 \cdot 10^{14}\right)^{2} .
\end{aligned}
$$

\section{EXPERIMENT}

The effect of specific parameters on the measurement accuracy can be tested using synthetic tests. Two different synthetic tests were used - Uniform Flow Test (UFT) and Couette Flow Test (CFT) [10. Uniform Flow Test simulates a velocity field with a constant displacement, Couette Flow Test simulates a velocity field with a constant velocity gradient within the whole Interrogation area. The mean displacement of the particles' deviation from the assumed value of the particle displacement is monitored during the tests. This deviation is denoted as a systematic error $\beta$. Another monitored parameter is the fluctuation of the displacement deviation around the mean value of the assumed displacement, which is denoted as a random error $\delta$. Sum of these errors is the total error of $\sigma$ the measured displacement. According to [11], mentioned 


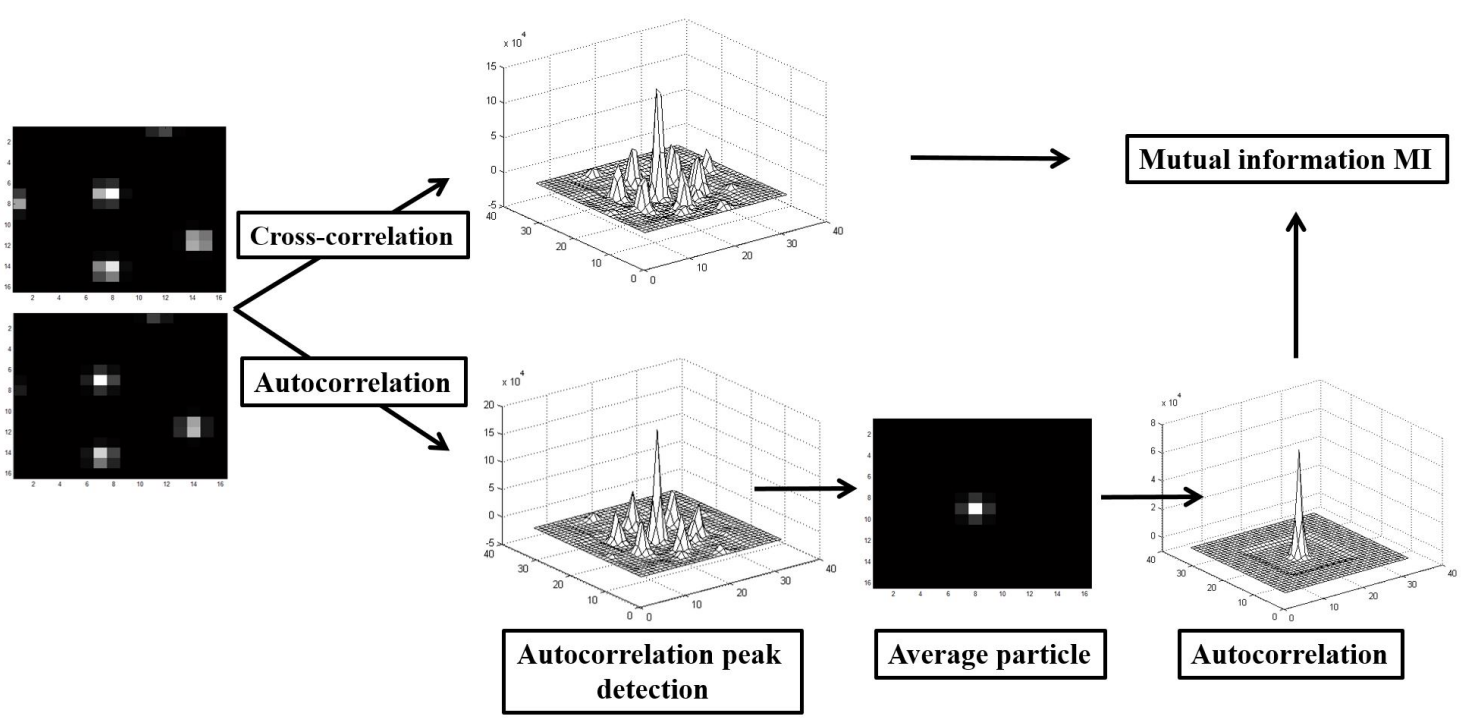

FIGURE 2. Schematic illustration of procedure of calculation metric MI [9].

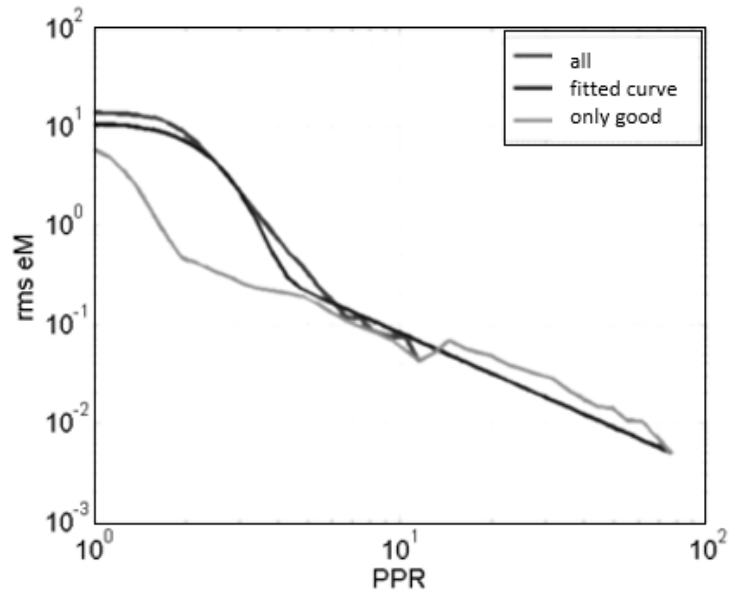

FIgURE 3. Root mean square error dependency on metric PPR [8].

errors are defined as follows:

$$
\begin{gathered}
\beta=u-\bar{u}, \quad \delta=\sqrt{\frac{1}{N} \sum_{i=1}^{N}\left(u_{i}-\bar{u}\right)^{2}}, \\
\delta=\sqrt{\frac{1}{N} \sum_{i=1}^{N}\left(u_{i}-u\right)^{2}}
\end{gathered}
$$

where $u$ is the value of the assumed displacement of the particle, $\bar{u}$ is the measured mean value of the displacement and $u_{i}$ is the measured displacement. When generating synthetic data, it is important to ensure that the synthetic data are generated correctly. An excellent guideline for a proper data generation can be found in [4, 12. In our work, the Standard cross-correlation (SCC) method was used to process the results of the synthetic tests. The resulting dependency between the specific value of the corrected metric and the measurement accuracy is applicable only to the SCC algorithm. A detailed description and results of the synthetic tests can be found in [13. An example of the results of both synthetic tests are shown in Figure 4

\section{Corrected metric}

Although the authors of the presented functions have done a great job, the use of these functions is only indicative. The metric PPR is not considering the effect of the number of the particles inside the interrogation area, the diameter of the particles and the displacement measured in the last iteration on the total error of the measurement. Also, the usefulness of (3) is arguable, because this dependency is mostly monitoring the set of the data of apparently wrongly measured vectors and not just correctly measured data. For this reason, it was necessary to define a new metric to address these mentioned shortcomings. At first, the new metric was corrected by the measured displacement in the last iteration, and also by the number of the particles inside the interrogation area IA. When defining the new metric, it is also necessary to consider the velocity gradient inside the IA. The value of the velocity gradient is defined as the ratio of the maximum difference of the displacement inside the IA and the diameter of the particle. The corrected metric $\mathrm{PPR}_{\Delta, \mathrm{MI}, \mathrm{GR}}$ can be formulated in the following form:

$$
\begin{aligned}
& \mathrm{PPR}_{\Delta, \mathrm{MI}, \mathrm{GR}} \\
& \begin{array}{c}
=\frac{\mathrm{PPR}-1}{0.01+\sqrt{\Delta x^{2}+\Delta y^{2}} \mathrm{MI}^{0.42}}+. \\
+\operatorname{sgn}(\mathrm{CR}) 4 \exp \left(\frac{\mathrm{MI}}{20}\right) \exp (\mathrm{GR})^{-1}
\end{array}
\end{aligned}
$$

To evaluate the dependency of the corrected metric $\mathrm{PPR}_{\Delta, \mathrm{MI}, \mathrm{GR}}$ on the total error of the measurement, 

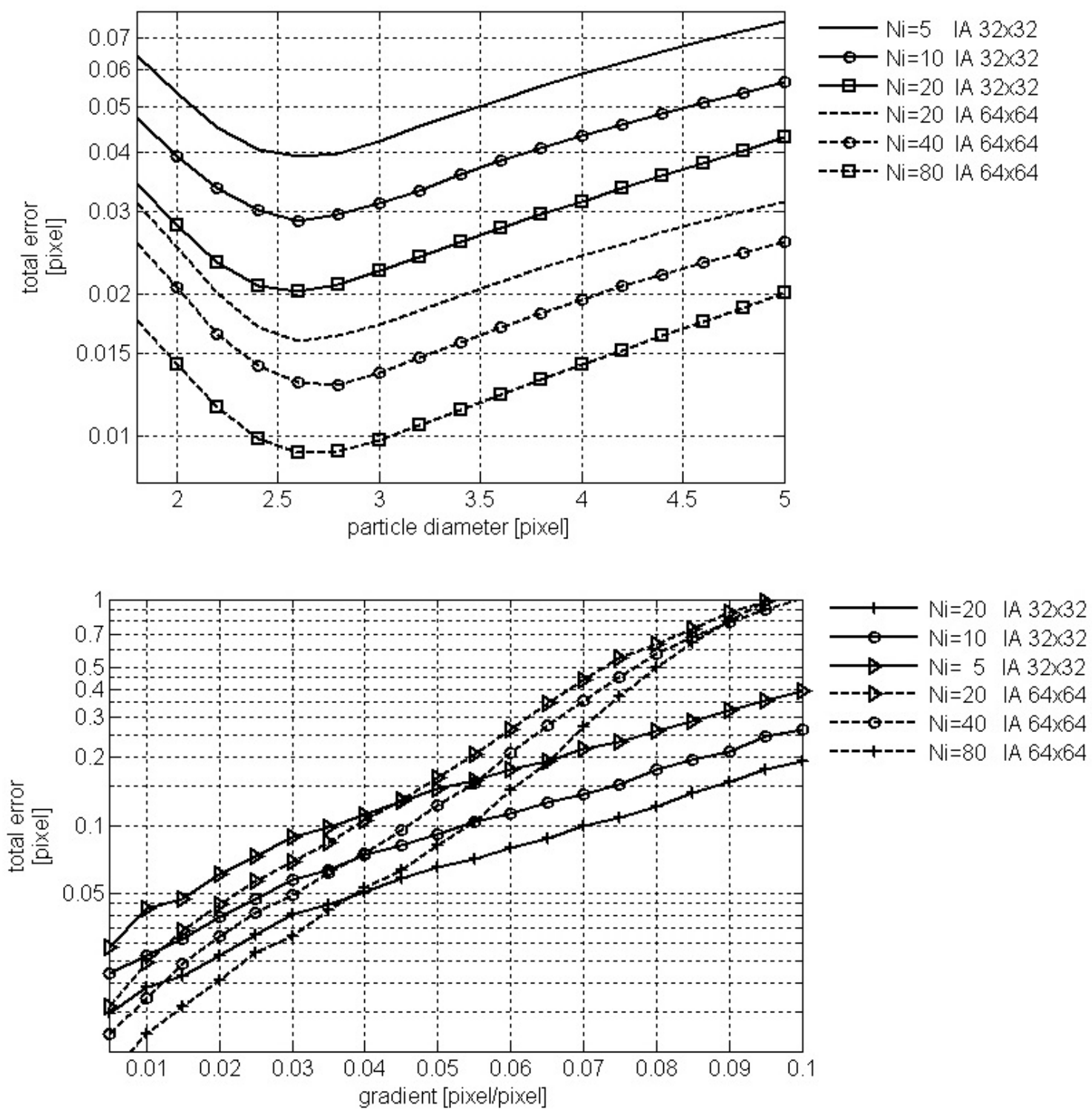

Figure 4. Example of the results of the synthetic tests - UFT (top), CFT (bottom) [13.

the synthetic tests mentioned in Section 2 were performed for several numbers of particles inside the IA and several values of the velocity gradient inside the IA. Based on these tests, a new definition of the measurement error dependency on the corrected metric was determined and formulated in the following form:

$$
\begin{aligned}
\sigma^{2}=\left(-1.5 \exp \left(-\mathrm{PPR}_{\Delta, \mathrm{MI}, \mathrm{GR}}^{2}\right)\right)^{2} & \\
& +0.8\left(\mathrm{PPR}_{\Delta, \mathrm{MI}, \mathrm{GR}}^{-1.1}\right)^{2} .
\end{aligned}
$$

The final graph showing the dependency of the total error of the measurement on the corrected metrics is shown in Figure 5

\section{Conclusions}

This work deals with the evaluation of a measurement accuracy based on the detection of the ratio of the primary and the secondary signal peak - the
PPR and its correction, considering other parameters influencing the measurement accuracy, by using the Particle Image Velocimetry method - PIV. Among other things, a procedure how to determine the number of the particles inside the IA is listed in this work. To define the corrected metric, a velocity gradient inside the IA was determined using the diameter of the particles and not, as it is usual, using the size of the edge of the IA. The corrected metric $\mathrm{PPR}_{\Delta, \mathrm{MI}, \mathrm{GR}}$ is introduced as well as its dependency on the total error of the measurement.

\section{LIST OF SYMBOLS}

$\Delta_{x}$ Horizontal displacement [pixel]

$\Delta_{y}$ Vertical displacement [pixel]

$A$ Coefficient of the correct value of displacement [1]

$B$ Exponent [1]

$C$ Minimum deviation of measurement [1] 


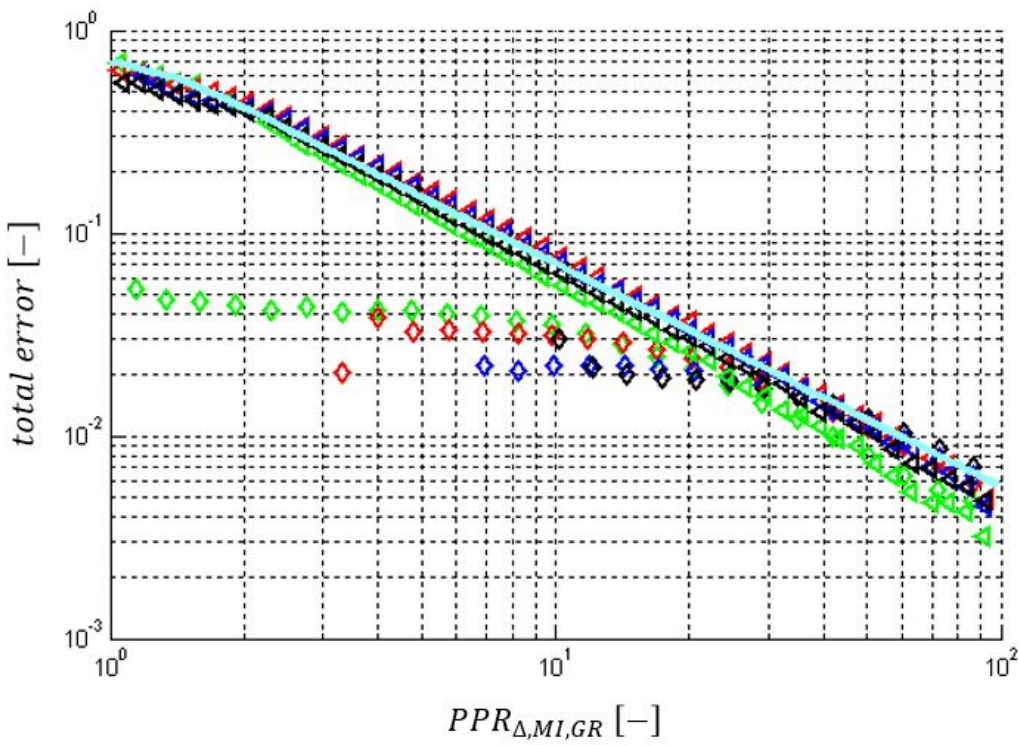

5 particles $32 \times 32$ CFT

10 particles $32 \times 32$ CFT

20 particles $32 \times 32$ CFT

30 particles $32 \times 32$ CFT

$4 \quad 5$ particles $32 \times 32$ UFT

$\triangleleft 10$ particles $32 \times 32$ UFT

$\triangleleft 20$ particles $32 \times 32$ UFT

$\triangleleft 30$ particles $32 \times 32$ UFT

Approximation

Figure 5. Dependency of the corrected metric $\mathrm{PPR}_{\Delta, \mathrm{MI}, \mathrm{GR}}$ on the total error of the measurement. Results of synthetic tests UFT and CFT for four different numbers of the particles. Velocity gradient inside IA was between the values 0 to 1.5 .

$D_{i} \quad$ Particle diameter [pixel]

$F_{\Delta}$ Velocity gradient [1]

$F_{\text {I }} \quad$ Number of lost pairs [1]

GR Velocity gradient [1]

$M$ Coefficient of total error of measurement [1]

MI Mutual information [1]

$N$ Minimum value of specific metric [1]

$N_{\text {I }} \quad$ Number of particles [1]

PCE Peak to correlation energy [1]

PPR Primary peak ratio [1]

$\mathrm{PPR}_{\Delta, \mathrm{MI}, \mathrm{GR}}$ Corrected Primary peak ratio [1]

PRMSR Peak to root-mean-square ratio [1]

$R_{\mathrm{C}} \quad$ Cross-correlation of noise [1]

$R_{\mathrm{D}}$ Cross-correlation of signal [1]

$R_{\mathrm{F}} \quad$ Cross-correlation of signal and noise [1]

$R_{\mathrm{S}}$ Cross-correlation of particle images [1]

$S$ Coefficient of real value of metric [1]

$u$ Assumed displacement [1]

$u_{i}$ Measured displacement [1]

$\bar{u} \quad$ Measured mean displacement [1]

$\beta \quad$ Systematic error [pixel]

$\delta$ Random error [pixel]

$\sigma$ Total error [pixel]

$\Phi \quad$ General metric value [1]

IA Interrogation area

CFT Couette Flow test

PIV Particle Image Velocimetry

SCC Standard Cross-correlation

UFT Uniform Flow test

\section{ACKNOWLEDGEMENTS}

Technological Agency, Czech Republic, program Center of Competence, project \#TE01020020 Josef Božek Competence Centre for Automotive Industry, The ministry of education, Youth and Sports program NPU I (LO), project \#LO1311, Development of Vehicle Centre of Sustainable Mobility. This support is gratefully acknowledged.

\section{REFERENCES}

[1] R. J. Adrian and C. Yao, "Pulsed Laser technique application to liquid and gaseous flows and the scattering power of seed materials," Applied optics, vol. 24, pp. 44-52, 1985. DOI:10.1364/AO.24.000044

[2] J. Westerweel, D. Dabiri and M. Gharib, "The Effect of a Discrete window offset on the Accuracy of Cross-Correlation Analysis of Digital PIV Recordings," Experiment in Fluids, vol. 23, pp. 20-28, 1997. DOI:10.1007/s003480050082

[3] R. J. Adrian, "Image shifting technique to resolve directional ambiguity in double-pulsed velocimetry," Applied Optics, vol. 25, no. 21, pp. 3855-3858, 1986. DOI:10.1364/AO.25.003855

[4] J. Westerweel, Digital Particle Image Velocimetry: Theory and Application, Delft: Delft University Press, 1993.

https://repository.tudelft.nl/islandora/object/ uuid:85455914-6629-4421-8c77-27cc44e771ed? collection=research [2018-06-17].

[5] J. Novotný, Analýza faktorů ovlivňujících vyhodnocení posunutí signálu při měření metodou Particle Image Velocimetry, Habilitační práce, Praha, 2016.

[6] J. Charonko and P. Vlachos, "Estimation of uncertainity bounds for individual particle image velocimetry measurements from cross-correlation peak ratio," Measurement Science and Technology, vol. 24, no. 6, 2013. DOI:10.1115/fedsm2012-72475

[7] A. Eckstein and P. Vlachos, "Digital Particle Image Velocimetry (DPIV) robust phase correlation," Measurement Science and Technology, vol. 20, no. 5, 2009. DOI:10.1088/0957-0233/20/5/055401 
[8] Z. Xue, J. Charonko and P. Vlachos, "Signal-to-noise ratio, Error and Uncertainty of PIV measurement," in International Symposium on Particle Image

Velocimetry, Delft, The Netherlands, 2013, July 1-3. https://repository.tudelft.nl/islandora/object/ uuid:a6270b28-1132-4186-817e-259eef0e9d87/ datastream/OBJ [2018-06-17].

[9] J. Novotný and I. Machovská, "Corrected metric for uncertainity estimation methods in Particle Image Velocimetry," in 9th World Conference on Experimental Heat Transfer, Fluid Mechanics and Thermodynamics, Foz do Iguazu, 2017.

[10] M. Raffel, C. Willert and J. Komphenhaus, Particle Image Velocity, Berlin: Springer-Verlang, 2007. DOI:10.1007/978-3-662-03637-2
[11] T. Astarita and Cardone G., "Analysis of Interpolation Schemes for Image Deformation Metods in PIV," Experiments in Fluids, vol. 38, pp. 233-243, 2005. DOI:10.1007/s00348-004-0902-3

[12] K. Okamoto, Nishio T., T. Saga and T. Kobayashi, "Standard Images for Particle Image Velocimetry," Measurement Sicence and Technology, vol. 11, pp. 685-691, 2000. DOI:10.1088/0957-0233/11/6/311

[13] J. Novotny, "Influence of Data Quality on Piv Measurement Accuracy," Journal of Flow Visualization and Image Processing, vol. 19, pp. 215-230, 2012. DOI:10.1615/jflowvisimageproc.2013003304 